\section{Insider dealing}

W. KRÄTSCHMER, R. Smalley and their colleagues are getting into buckminsterfullerene like no others. The first group (T. Weiske et al. Angew. Chemie Int. Edn. Engl. 30, 884-886; 1991) have invented what they term 'endohedral' chemistry by incorporating helium and neon atoms into the interiors of the carbon shells through high-energy collisions. Pairs of carbon atoms are ablated from the $\mathrm{C}_{60}$ clusters in the collisions, apparently as the inert atoms punch their way in. Smalley and colleagues (T. Guo et al. J. Am. chem. Soc. 95, 4948-4950; 1991), not delving so deeply, find it is possible to substitute boron atoms for individual carbon atoms in buckminsterfullerene's shell to produce a Lewis acid that attaches ammonia molecules to its exterior. In the meantime, M. Saunders (Science 253, 330-331; 1991) calculates that the fully hydrogenated fullerane $\mathrm{C}_{60} \mathrm{H}_{60}$ is furry on the outside and on the inside, the carbon - carbon bond strain being minimized if several of the hydrogen atoms find their way into the molecule's interior.

\section{Hunting the gene}

IT has been eight years since the dramatic mapping of the gene for the neurodegenerative disorder Huntington's disease to chromosome 4 , yet attempts to assign the gene more accurately to one of two 'candidate' regions near the telomere have been confounded. Concentrating on the more proximal region, G. P. Bates and colleagues have used a combination of genetic and physical mapping methods to show that the distance between two DNA markers thought to flank the Huntington's gene is a mere 2.5 megabases (Am. J. hum. Genet. 49, 7-16; 1991). Although it may still take a considerable time before the gene itself is identified, the signs are encouraging that the boundaries for the search have finally been delineated.

\section{Split decision}

NEW limits on exotic particles, calculated by J. Gratsias, R. J. Scherrer and D. N. Spergel (Phys. Lett. B262, 298-302; 1991) close off, or at least constrict, a favoured let out for cosmologists whose Big Bang models don't quite predict the observed Universe. The idea is that short-lived, massive particles existing immediately after the Big Bang altered the subsequent evolution of the Universe, but are conveniently no longer around. They are usually disposed of by being made to decay into energetic neutrinos, which barely interact with other material in the Universe. But Gratsias et al. show that annihilation of decay neutrinos on cosmic background neutrinos creates photons that would split the light nuclei synthesized in the Big Bang. Solving one problem, it seems, simply creates another. (packing of hydrophobic faces of amphiphilic helices). Numerous studies of $\alpha$-helix formation in oligopeptides have been designed to clarify the situation, using variants of these natural sequences, and synthetic sequences designed for systematic variation in aminoacid composition and sequence.

Anyone wishing to participate will have to carry out the following steps. (1) Purify your material; (2) find conditions in which it is at least partially $\alpha$ helical, and prove its structure by circular dichroism or nuclear magnetic resonance (preferably both); (3) measure its fractional helix content in a variety of conditions; (4) verify that the measurements are consistent with the Zimm-Bragg theory; and (5) derive values of $\sigma$ and $s$ by fitting the data to available equations. Then compare your results with those from earlier work and from the new studies $^{1-4}$

The paper by Chakrabartty et al. ${ }^{2}$, the latest in a series of reports from Baldwin's group, is a study of an alanine-rich reference peptide and 12 related peptides with Gly substituted for each of the Ala residues. Formation of $\alpha$ helices at $273 \mathrm{~K}$ and $\mathrm{pH} 7$ was measured by circular dichroism, and the results fitted to the theory, with the result $\sigma=0.0029$, $s$ (Gly) $\sim 0.015$ and $s$ (Ala) is no less than 1.5. As in several other studies, the value for Ala is much higher than that of Scheraga and coworkers ${ }^{7}$.

Kemp and colleagues ${ }^{1}$, taking a different approach, have designed an $\mathrm{N}$ terminal 'template' to initiate and 'report' helical structure in an attached oligopeptide. The template, a bridged derivative of the dipeptide Pro-Pro, is a kind of 'flip-flop', in a conformational equilibrium between one state that can form hydrogen bonds to an attached peptide in the $\alpha$-helical conformation, and another state that cannot form such hydrogen bonds. Thus $\alpha$-helix formation in the attached peptide will be reflected in a shift in the equilibrium of the template, detectable by NMR. The authors apply this method to alanine-rich oligopeptides at $298 \mathrm{~K}$ and $\mathrm{pH} 1-2$. No circular dichroism data are presented, and I must admit that I would be happier with a more rigorous demonstration that no conformations of the attached peptide except the $\alpha$ helix can interact with the template to affect its equilibrium. From the shifts in the equilibrium of the template, the authors infer values of $s($ Ala $) \sim 1.0$ and $s$ (Gly) $\sim 0.3-0.6$, consistent with the results of Scheraga and coworkers ${ }^{7}$ but not with those of Chakrabartty et al. ${ }^{2}$.

In another application of the hostguest method, Kallenbach and coworkers ${ }^{3,4}$ studied $\alpha$-helix formation in oligopeptides containing the sequence $\mathrm{Glu}_{4}-\mathrm{Lys}_{4}-\mathrm{X}_{3}-\mathrm{Glu}_{4}-\mathrm{Lys}_{4}$ in which the
Glu $_{4}$ Lys $_{4}$ blocks provided solubility and stabilized the $\alpha$-helical conformation through salt bridges along the helix surface and the guest residue $\mathrm{X}$ included natural amino acids ${ }^{3}$ and unnatural ones containing alkyl groups with various branching patterns, to test the effect on helix stability of steric constraints on side-chain conformation ${ }^{3,4}$. Circulardichroism spectra at $277 \mathrm{~K}$ and $\mathrm{pH} 7$ are reported. For natural amino acids, the conformational preference parameters are similar but not identical to those determined by Baldwin and coworkers, and correlate well with other previous results ${ }^{5}$. The unnatural amino acids suggest that the branching pattern of alkyl side-chains has a very important effect on $\alpha$-helix stability, as a result of steric interactions between side chain and main chain. Baldwin and coworkers have made similar observations ${ }^{8,9}$.

What then can the spectator conclude? There is consensus that the effects of sequence context and tertiary interactions strongly influence helix formation. Yet most authors insist (occasionally perhaps protesting too much) that there must be a unique set of numbers that expresses the ideal helix-forming tendency of amino acids in monomeric oligopeptides, in the absence of complicating effects such as explicit side-chain interactions and variations in conditions of solvent and temperature. Those who accept this second premise will note the general but imperfect qualitative agreement about relative helix-promoting tendencies of amino acids, and will await additional experiments that, if the premise is true, must ultimately reconcile the differences among reported values of Zimm-Bragg parameters. Others may wonder whether so few parameters can really do so much for so many variations and combinations of amino acids under different conditions of solvent and temperature, and will find it possible to accept that many if not all of the results can be valid, if only for the systems from which they were derived.

Arthur M. Lesk is in the Department of Haematology, University of Cambridge, Addenbrooke's Hospital, Cambridge CB2 2QL, UK.

1. Kemp, D. S., Boyd, J. G. \& Muendel, C. C. Nature 352 451-454 (1991)

2. Chakrabartty, A., Schellman, J. A. \& Baldwin, R. L. Nature 351, 586-588 (1991)

3. Lyu, P. C., Liff, M. I. Marky, L. A. \& Kallenbach, N. R. Science 250, 669-673 (1990)

4. Lyu, P. C., Sherman, J. C., Chen, A. \& Kallenbach, N. R. Proc. natn. Acad. Sci. U.S.A. 88, 5317-5320 (1991).

5. O'Neil, K. T. \& DeGrado, W. F. Science 250, 646-651 (1990).

6. Zimm, B. H. \& Bragg, J. K. J. chem. Phys. 31, 526-535 (1959)

7. Sueki, M., Lee, S., Powers, S. P., Denton, J. B., Konishi, Y. \& Scheraga, H. A. Macromolecules 17, 148-155 (1984).

8. Padmanabhan, S., Marqusee, S., Ridgeway, T., Laue, T. M. \& Baldwin, R. L. Nature 344, 268-270 (1990) 9. Padmanabhan, S. \& Baldwin, R. L. J. molec. Biol. 219 135-137 (1991) 